\title{
The basis of dimension-six operators in the Standard Model
}

\author{
Mateusz Iskrzyński* \\ Institute of Theoretical Physics, University of Warsaw, \\ Hoża 69, PL-00-681 Warsaw, Poland. \\ E-mail: mateusz.iskrzynski@fuw.edu.pl
}

\begin{abstract}
When one considers an effective low-energy theory built upon the basis of the Standard Model (i.e. posessing its fields and symmetries), higher dimensional interaction terms appear in the Lagrangian. Dimension-six terms have been enumerated in the classical article by Buchmüller and Wyler [1]. Although redundance of some of those operators has been already noted in the literature, the first complete list has been published in the article [2], where we performed their classification once again from the outset. Assuming baryon number conservation, we found $15+19+25=59$ independent operators (barring flavour structure and Hermitian conjugations), as compared to $16+35+29=80$ in Ref. [1]. The three summed numbers refer to operators containing 0, 2 and 4 fermion fields. If the assumption of baryon number conservation is relaxed, 5 new operators arise in the four-fermion sector.
\end{abstract}

Proceedings of the Corfu Summer Institute 2011 "School and Workshops on Elementary Particle Physics and Gravity"

September 4-18, 2011

Corfu, Greece

${ }^{*}$ This publication follows the article by Bohdan Grzadkowski, Mateusz Iskrzyński, Mikolaj Misiak, Janusz Rosiek "Dimension-Six Terms in the Standard Model Lagrangian", JHEP 10 (2010) 85 


\section{Introduction}

It is commonly believed, that the the Standard Model (SM) of strong and electroweak interactions is an effective theory that is valid up to certain energy scale $\Lambda$. The theory embedding it would have to incorporate all the SM degrees of freedom and, if a gauge theory, posess an internal symmetry containing $S U(3)_{C} \times S U(2)_{L} \times U(1)_{Y}$ of the $S M$, whereas its predictions should reduce at low energies to those of the SM.

Typically for theories that are considered, reduction to the SM at low energies proceeds via decoupling of heavy particles with masses of order $\Lambda$ or larger. Such a decoupling at the perturbative level is described by the Appelquist-Carazzone postulate [3]. It leads to appearance of higher-dimensional operators in the Lagrangian of considered effective theory that are suppressed by powers of $\Lambda$

$$
\mathscr{L}_{\mathrm{S} M}=\mathscr{L}_{\mathrm{S} M}^{(4)}+\frac{1}{\Lambda} \sum_{k} C_{k}^{(5)} Q_{k}^{(5)}+\frac{1}{\Lambda^{2}} \sum_{k} C_{k}^{(6)} Q_{k}^{(6)}+\mathscr{O}\left(\frac{1}{\Lambda^{3}}\right)
$$

where $\mathscr{L}_{\mathrm{S} M}^{(4)}$ is the usual "renormalizable" part of the SM Lagrangian, containing only dimensiontwo and -four operators. ${ }^{1}$ In the remaining terms, $Q_{k}^{(n)}$ denote dimension- $n$ operators, and $C_{k}^{(n)}$ stand for the corresponding dimensionless coupling constants (Wilson coefficients). Once the underlying high-energy theory is specified, all the coefficients $C_{k}^{(n)}$ can be determined by integrating out the heavy fields. In practice however, one rather determines them, requiring that both theories give the same values of Green's functions.

The goal we achieved was to find a complete set of independent operators of dimension 5 and 6 that are built out of the SM fields and are consistent with the SM gauge symmetries. We did not rely on the original analysis of such operators by Buchmüller and Wyler [1] and performed the full classification. The main reason for repeating the analysis was the fact that many linear combinations of operators listed in Ref. [1] vanish by the Equations Of Motion (EOMs). Such operators are redundant, i.e. they give no contribution to on-shell matrix elements, both in perturbation theory (to all orders) and beyond [4]. Although the presence of several EOM-vanishing combinations in Ref. [1] has been already pointed out in the literature [14, 13, 15], no updated complete list has been published to date. Our final operator basis differs from Ref. [1] also in the four-fermion sector where the EOMs play no role.

\section{Notation and conventions}

The SM matter content is summarized in Tab. 1 with isospin, colour, and generation indices denoted by $j=1,2, \alpha=1,2,3$, and $p=1,2,3$, respectively. Chirality indices $(L, R)$ of the fermion fields will be skipped in what follows. Complex conjugate of the Higgs field will always occur either as $\varphi^{\dagger}$ or $\widetilde{\varphi}$, where $\widetilde{\varphi}^{j}=\varepsilon_{j k}\left(\varphi^{k}\right)^{\star}$, and $\varepsilon_{j k}$ is totally antisymmetric with $\varepsilon_{12}=+1$.

\footnotetext{
${ }^{1}$ Canonical dimensions of operators are determined from the field contents alone, excluding possible dimensionful coupling constants. The only dimension-two operator in $\mathscr{L}_{\mathrm{S} M}^{(4)}$ is $\varphi^{\dagger} \varphi$ in the Higgs mass term.
} 


\begin{tabular}{|c|ccccc|c|}
\hline & \multicolumn{5}{|c|}{ fermions } & scalars \\
\hline field & $l_{L p}^{j}$ & $e_{R p}$ & $q_{L p}^{\alpha j}$ & $u_{R p}^{\alpha}$ & $d_{R p}^{\alpha}$ & $\varphi^{j}$ \\
\hline hypercharge $Y$ & $-\frac{1}{2}$ & -1 & $\frac{1}{6}$ & $\frac{2}{3}$ & $-\frac{1}{3}$ & $\frac{1}{2}$ \\
\hline
\end{tabular}

Table 1: The SM matter content

The well-known expression for $\mathscr{L}_{\mathrm{SM}}^{(4)}$ before Spontaneous Symmetry Breakdown (SSB) reads

$$
\begin{aligned}
\mathscr{L}_{\mathrm{S} M}^{(4)} & =-\frac{1}{4} G_{\mu v}^{A} G^{A \mu v}-\frac{1}{4} W_{\mu v}^{I} W^{I \mu v}-\frac{1}{4} B_{\mu v} B^{\mu v}+\left(D_{\mu} \varphi\right)^{\dagger}\left(D^{\mu} \varphi\right)+m^{2} \varphi^{\dagger} \varphi-\frac{1}{2} \lambda\left(\varphi^{\dagger} \varphi\right)^{2} \\
& +i(\bar{l} \not \supset l+\bar{e} \not D e+\bar{q} \not D q+\bar{u} \not D u+\bar{d} \not D d)-\left(\bar{l} \Gamma_{e} e \varphi+\bar{q} \Gamma_{u} u \widetilde{\varphi}+\bar{q} \Gamma_{d} d \varphi+\text { h.c. }\right)
\end{aligned}
$$

where the Yukawa couplings $\Gamma_{e, u, d}$ are matrices in the generation space. We shall not consider SSB in this paper. Our sign convention for covariant derivatives is exemplified by

$$
D_{\mu} q=\left(\partial_{\mu}+i g_{s} T^{A} G_{\mu}^{A}+i g S^{I} W_{\mu}^{I}+i g^{\prime} Y_{q} B_{\mu}\right) q
$$

Here, $T^{A}=\frac{1}{2} \lambda^{A}$ and $S^{I}=\frac{1}{2} \tau^{I}$ are the $S U(3)$ and $S U(2)$ generators, while $\lambda^{A}$ and $\tau^{I}$ are the GellMann and Pauli matrices, respectively. All the hypercharges $Y$ have been listed in Tab. 1.

It is useful to define Hermitian derivative terms that contain $\varphi \overleftarrow{D_{\mu}} \varphi \equiv\left(D_{\mu} \varphi\right)^{\dagger} \varphi$ as follows:

$$
\varphi^{\dagger} \stackrel{\leftrightarrow}{D_{\mu}} \varphi \equiv i \varphi^{\dagger}\left(D_{\mu}-\overleftarrow{D_{\mu}}\right) \varphi \quad \text { and } \quad \varphi^{\dagger} \stackrel{\leftrightarrow}{D_{\mu}^{I}} \varphi \equiv i \varphi^{\dagger}\left(\tau^{I} D_{\mu}-\overleftarrow{D_{\mu}} \tau^{I}\right) \varphi
$$

The gauge field strength tensors and their covariant derivatives read

$$
\begin{array}{rlrl}
G_{\mu \nu}^{A} & =\partial_{\mu} G_{v}^{A}-\partial_{\nu} G_{\mu}^{A}-g_{s} f^{A B C} G_{\mu}^{B} G_{v}^{C}, & \left(D_{\rho} G_{\mu \nu}\right)^{A}=\partial_{\rho} G_{\mu \nu}^{A}-g_{s} f^{A B C} G_{\rho}^{B} G_{\mu \nu}^{C}, \\
W_{\mu \nu}^{I}=\partial_{\mu} W_{v}^{I}-\partial_{v} W_{\mu}^{I}-g \varepsilon^{I J K} W_{\mu}^{J} W_{v}^{K}, & \left(D_{\rho} W_{\mu \nu}\right)^{I}=\partial_{\rho} W_{\mu \nu}^{I}-g \varepsilon^{I J K} W_{\rho}^{J} W_{\mu \nu}^{K}, \\
B_{\mu v}=\partial_{\mu} B_{v}-\partial_{v} B_{\mu}, & D_{\rho} B_{\mu \nu}=\partial_{\rho} B_{\mu \nu} .
\end{array}
$$

Dual tensors are defined by $\widetilde{X}_{\mu v}=\frac{1}{2} \varepsilon_{\mu \nu \rho \sigma} X^{\rho \sigma}\left(\varepsilon_{0123}=+1\right)$, where $X$ stands for $G^{A}, W^{I}$ or $B$.

The fermion kinetic terms in $\mathscr{L}_{\mathrm{S} M}^{(4)}$ are Hermitian up to total derivatives, i.e. $i \bar{\psi} \not D \psi-$ h.c. $=\partial_{\mu}\left(\bar{\psi} \gamma^{\mu} \psi\right)$. Total derivatives of gauge-invariant objects in $\mathscr{L}_{\mathrm{S} M}$ are skipped throughout the paper, as they give no physical effects. At the dimension-five and -six levels, we encounter no gauge-invariant operators that are built out of non-abelian gauge fields only, and equal to total derivatives of gauge-variant objects. At the dimension-four level, the two possible such terms $\widetilde{G}_{\mu \nu}^{A} G^{A \mu \nu}=4 \varepsilon^{\mu \nu \rho \sigma} \partial_{\mu}\left(G_{v}^{A} \partial_{\rho} G_{\sigma}^{A}-\frac{1}{3} g_{s} f^{A B C} G_{v}^{A} G_{\rho}^{B} G_{\sigma}^{C}\right)$ and the analogous $\widetilde{W}_{\mu \nu}^{I} W^{I \mu \nu}$ should be understood as implicitly present on the r.h.s of Eq. (2.1). They leave the Feynman rules and EOMs unaffected, showing up in topological quantum effects only [5]. 


\begin{tabular}{|c|c|c|c|c|c|}
\hline \multicolumn{2}{|r|}{$X^{3}$} & \multicolumn{2}{|r|}{$\varphi^{6}$ and $\varphi^{4} D^{2}$} & \multicolumn{2}{|r|}{$\psi^{2} \varphi^{3}$} \\
\hline$Q_{G}$ & $f^{A B C} G_{\mu}^{A v} G_{v}^{B \rho} G_{\rho}^{C \mu}$ & $Q_{\varphi}$ & $\left(\varphi^{\dagger} \varphi\right)^{3}$ & $Q_{e \varphi}$ & $\left(\varphi^{\dagger} \varphi\right)\left(\bar{l}_{p} e_{r} \varphi\right)$ \\
\hline$Q_{\widetilde{G}}$ & $f^{A B C} \widetilde{G}_{\mu}^{A v} G_{v}^{B \rho} G_{\rho}^{C \mu}$ & $Q_{\varphi \square}$ & $\left(\varphi^{\dagger} \varphi\right) \square\left(\varphi^{\dagger} \varphi\right)$ & $Q_{u \varphi}$ & $\left(\varphi^{\dagger} \varphi\right)\left(\bar{q}_{p} u_{r} \widetilde{\varphi}\right)$ \\
\hline$Q_{W}$ & $\varepsilon^{I J K} W_{\mu}^{I v} W_{v}^{J \rho} W_{\rho}^{K \mu}$ & $Q_{\varphi D}$ & $\left(\varphi^{\dagger} D^{\mu} \varphi\right)^{\star}\left(\varphi^{\dagger} D_{\mu} \varphi\right)$ & $Q_{d \varphi}$ & $\left(\varphi^{\dagger} \varphi\right)\left(\bar{q}_{p} d_{r} \varphi\right)$ \\
\hline$Q_{\widetilde{W}}$ & $\varepsilon^{I J K} \widetilde{W}_{\mu}^{I v} W_{v}^{J \rho} W_{\rho}^{K \mu}$ & & & & \\
\hline \multicolumn{2}{|r|}{$X^{2} \varphi^{2}$} & \multicolumn{2}{|r|}{$\psi^{2} X \varphi$} & \multicolumn{2}{|r|}{$\psi^{2} \varphi^{2} D$} \\
\hline$Q_{\varphi G}$ & $\varphi^{\dagger} \varphi G_{\mu \nu}^{A} G^{A \mu \nu}$ & $Q_{e W}$ & $\left(\bar{l}_{p} \sigma^{\mu v} e_{r}\right) \tau^{I} \varphi W_{\mu v}^{I}$ & $Q_{\varphi l}^{(1)}$ & $\left(\varphi^{\dagger}{\stackrel{\leftrightarrow}{D_{\mu}}}_{\varphi} \varphi\right)\left(\bar{l}_{p} \gamma^{\mu} l_{r}\right)$ \\
\hline$Q_{\varphi \widetilde{G}}$ & $\varphi^{\dagger} \varphi \widetilde{G}_{\mu \nu}^{A} G^{A \mu \nu}$ & $Q_{e B}$ & $\left(\bar{l}_{p} \sigma^{\mu v} e_{r}\right) \varphi B_{\mu v}$ & $Q_{\varphi l}^{(3)}$ & $\left(\varphi^{\dagger} \stackrel{\leftrightarrow}{D_{\mu}^{I}} \varphi\right)\left(\bar{l}_{p} \tau^{I} \gamma^{\mu} l_{r}\right)$ \\
\hline$Q_{\varphi W}$ & $\varphi^{\dagger} \varphi W_{\mu \nu}^{I} W^{I \mu \nu}$ & $Q_{u G}$ & $\left(\bar{q}_{p} \sigma^{\mu v} T^{A} u_{r}\right) \widetilde{\varphi} G_{\mu v}^{A}$ & $Q_{\varphi e}$ & $\left(\varphi^{\dagger} \stackrel{\leftrightarrow}{D_{\mu}} \varphi\right)\left(\bar{e}_{p} \gamma^{\mu} e_{r}\right)$ \\
\hline$Q_{\varphi \widetilde{W}}$ & $\varphi^{\dagger} \varphi \widetilde{W}_{\mu v}^{I} W^{I \mu \nu}$ & $Q_{u W}$ & $\left(\bar{q}_{p} \sigma^{\mu v} u_{r}\right) \tau^{I} \widetilde{\varphi} W_{\mu v}^{I}$ & $Q_{\varphi q}^{(1)}$ & $\left(\varphi^{\dagger} \stackrel{\leftrightarrow}{D}_{\mu} \varphi\right)\left(\bar{q}_{p} \gamma^{\mu} q_{r}\right)$ \\
\hline$Q_{\varphi B}$ & $\varphi^{\dagger} \varphi B_{\mu v} B^{\mu v}$ & $Q_{u B}$ & $\left(\bar{q}_{p} \sigma^{\mu v} u_{r}\right) \widetilde{\varphi} B_{\mu v}$ & $Q_{\varphi q}^{(3)}$ & $\left(\varphi^{\dagger} \overleftrightarrow{D_{\mu}^{I}} \varphi\right)\left(\bar{q}_{p} \tau^{I} \gamma^{\mu} q_{r}\right)$ \\
\hline$Q_{\varphi \widetilde{B}}$ & $\varphi^{\dagger} \varphi \widetilde{B}_{\mu v} B^{\mu v}$ & $Q_{d G}$ & $\left(\bar{q}_{p} \sigma^{\mu v} T^{A} d_{r}\right) \varphi G_{\mu v}^{A}$ & $Q_{\varphi u}$ & $\left(\varphi^{\dagger} \stackrel{\leftrightarrow}{D_{\mu}} \varphi\right)\left(\bar{u}_{p} \gamma^{\mu} u_{r}\right)$ \\
\hline$Q_{\varphi W B}$ & $\varphi^{\dagger} \tau^{I} \varphi W_{\mu v}^{I} B^{\mu \nu}$ & $Q_{d W}$ & $\left(\bar{q}_{p} \sigma^{\mu v} d_{r}\right) \tau^{I} \varphi W_{\mu v}^{I}$ & $Q_{\varphi d}$ & $\left(\varphi^{\dagger}{\stackrel{\leftrightarrow}{D_{\mu}}}_{\varphi}\right)\left(\bar{d}_{p} \gamma^{\mu} d_{r}\right)$ \\
\hline$Q_{\varphi \widetilde{W} B}$ & $\varphi^{\dagger} \tau^{I} \varphi \widetilde{W}_{\mu \nu}^{I} B^{\mu v}$ & $Q_{d B}$ & $\left(\bar{q}_{p} \sigma^{\mu v} d_{r}\right) \varphi B_{\mu v}$ & $Q_{\varphi \text { ud }}$ & $i\left(\widetilde{\varphi}^{\dagger} D_{\mu} \varphi\right)\left(\bar{u}_{p} \gamma^{\mu} d_{r}\right)$ \\
\hline
\end{tabular}

Table 2: Dimension-six operators other than the four-fermion ones.

\section{The complete set of dimension-five and -six operators}

This Section is devoted to presenting our final results for the basis of independent operators $Q_{n}^{(5)}$ and $Q_{n}^{(6)}$, meaning that no linear combination of them and their Hermitian conjugates is EOMvanishing up to total derivatives.

Imposing the SM gauge symmetry constraints on $Q_{n}^{(5)}$ leaves out just a single operator [6], up to Hermitian conjugation and flavour assignments. It reads

$$
Q_{v v}=\varepsilon_{j k} \varepsilon_{m n} \varphi^{j} \varphi^{m}\left(l_{p}^{k}\right)^{T} C l_{r}^{n} \equiv\left(\widetilde{\varphi}^{\dagger} l_{p}\right)^{T} C\left(\widetilde{\varphi}^{\dagger} l_{r}\right),
$$

where $C$ is the charge conjugation matrix. ${ }^{2} Q_{v v}$ violates the lepton number $L$. After the electroweak symmetry breaking, it generates neutrino masses and mixings. Neither $\mathscr{L}_{\mathrm{S} M}^{(4)}$ nor the dimensionsix terms can do the job. Thus, consistency of the SM (as defined by Eq. (1.1) and Tab. 1) with observations crucially depends on this dimension-five term.

All the independent dimension-six operators that are allowed by the SM gauge symmetries are listed in Tabs. 2 and 3. Their names in the left column of each block should be supplemented with generation indices of the fermion fields whenever necessary, e.g., $Q_{l q}^{(1)} \rightarrow Q_{l q}^{(1) p r s t}$. Dirac indices are always contracted within the brackets, and not displayed. The same is true for the isospin and

\footnotetext{
${ }^{2}$ In the Dirac representation $C=i \gamma^{2} \gamma^{0}$, with Bjorken and Drell[7] phase conventions.
} 


\begin{tabular}{|c|c|c|c|c|c|}
\hline \multicolumn{2}{|r|}{$(\bar{L} L)(\bar{L} L)$} & \multicolumn{2}{|r|}{$(\bar{R} R)(\bar{R} R)$} & \multicolumn{2}{|r|}{$(\bar{L} L)(\bar{R} R)$} \\
\hline$Q_{l l}$ & $\left(\bar{l}_{p} \gamma_{\mu} l_{r}\right)\left(\bar{l}_{s} \gamma^{\mu} l_{t}\right)$ & $Q_{e e}$ & $\left(\bar{e}_{p} \gamma_{\mu} e_{r}\right)\left(\bar{e}_{s} \gamma^{\mu} e_{t}\right)$ & & $\left(\bar{l}_{p} \gamma_{\mu} l_{r}\right)\left(\bar{e}_{s} \gamma^{\mu} e_{t}\right)$ \\
\hline$Q_{q q}^{(1)}$ & $\left(\bar{q}_{p} \gamma_{\mu} q_{r}\right)\left(\bar{q}_{s} \gamma^{\mu} q_{t}\right)$ & $Q_{\text {ии }}$ & $\left(\bar{u}_{p} \gamma_{\mu} u_{r}\right)\left(\bar{u}_{s} \gamma^{\mu} u_{t}\right)$ & $Q_{l u}$ & $\left(\bar{l}_{p} \gamma_{\mu} l_{r}\right)\left(\bar{u}_{s} \gamma^{\mu} u_{t}\right)$ \\
\hline$Q_{q q}^{(3)}$ & $\left(\bar{q}_{p} \gamma_{\mu} \tau^{I} q_{r}\right)\left(\bar{q}_{s} \gamma^{\mu} \tau^{I} q_{t}\right)$ & $Q_{d d}$ & $\left(\bar{d}_{p} \gamma_{\mu} d_{r}\right)\left(\bar{d}_{s} \gamma^{\mu} d_{t}\right)$ & $Q_{l d}$ & $\left(\bar{l}_{p} \gamma_{\mu} l_{r}\right)\left(\bar{d}_{s} \gamma^{\mu} d_{t}\right)$ \\
\hline$Q_{l q}^{(1)}$ & $\left(\bar{l}_{p} \gamma_{\mu} l_{r}\right)\left(\bar{q}_{s} \gamma^{\mu} q_{t}\right)$ & $Q_{e u}$ & $\left(\bar{e}_{p} \gamma_{\mu} e_{r}\right)\left(\bar{u}_{s} \gamma^{\mu} u_{t}\right)$ & $Q_{q e}$ & $\left(\bar{q}_{p} \gamma_{\mu} q_{r}\right)\left(\bar{e}_{s} \gamma^{\mu} e_{t}\right)$ \\
\hline \multirow[t]{4}{*}{$Q_{l q}^{(3)}$} & $\left(\bar{l}_{p} \gamma_{\mu} \tau^{I} l_{r}\right)\left(\bar{q}_{s} \gamma^{\mu} \tau^{I} q_{t}\right)$ & $Q_{e d}$ & $\left(\bar{e}_{p} \gamma_{\mu} e_{r}\right)\left(\bar{d}_{s} \gamma^{\mu} d_{t}\right)$ & $Q_{q u}^{(1)}$ & $\left(\bar{q}_{p} \gamma_{\mu} q_{r}\right)\left(\bar{u}_{s} \gamma^{\mu} u_{t}\right)$ \\
\hline & & $Q_{u d}^{(1)}$ & $\left(\bar{u}_{p} \gamma_{\mu} u_{r}\right)\left(\bar{d}_{s} \gamma^{\mu} d_{t}\right)$ & $Q_{q u}^{(8)}$ & $\left(\bar{q}_{p} \gamma_{\mu} T^{A} q_{r}\right)\left(\bar{u}_{s} \gamma^{\mu} T^{A} u_{t}\right)$ \\
\hline & & $Q_{u d}^{(8)}$ & $\left(\bar{u}_{p} \gamma_{\mu} T^{A} u_{r}\right)\left(\bar{d}_{s} \gamma^{\mu} T^{A} d_{t}\right)$ & $Q_{q d}^{(1)}$ & $\left(\bar{q}_{p} \gamma_{\mu} q_{r}\right)\left(\bar{d}_{s} \gamma^{\mu} d_{t}\right)$ \\
\hline & & & & $Q_{q d}^{(8)}$ & $\left(\bar{q}_{p} \gamma_{\mu} T^{A} q_{r}\right)\left(\bar{d}_{s} \gamma^{\mu} T^{A} d_{t}\right)$ \\
\hline \multicolumn{2}{|c|}{$(\bar{L} R)(\bar{R} L)$ and $(\bar{L} R)(\bar{L} R)$} & \multicolumn{4}{|c|}{$B$-violating } \\
\hline$Q_{l e d q}$ & $\left(\bar{l}_{p}^{j} e_{r}\right)\left(\bar{d}_{s} q_{t}^{j}\right)$ & $Q_{d u q}$ & $\varepsilon^{\alpha \beta \gamma} \varepsilon_{j k}\left[\left(d_{p}^{\alpha}\right.\right.$ & $\left.{ }^{T} C u_{r}^{\beta}\right]$ & $\left.\left(q_{s}^{\gamma j}\right)^{T} C l_{t}^{k}\right]$ \\
\hline$Q_{q u q d}^{(1)}$ & $\left(\bar{q}_{p}^{j} u_{r}\right) \varepsilon_{j k}\left(\bar{q}_{s}^{k} d_{t}\right)$ & $Q_{q q u}$ & $\varepsilon^{\alpha \beta \gamma} \varepsilon_{j k}\left[\left(q_{p}^{\alpha}\right.\right.$ & ${ }^{T} C q_{r}^{\beta k}$ & {$\left[\left(u_{S}^{\gamma}\right)^{T} C e_{t}\right]$} \\
\hline$Q_{q u q d}^{(8)}$ & $\left(\bar{q}_{p}^{j} T^{A} u_{r}\right) \varepsilon_{j k}\left(\bar{q}_{s}^{k} T^{A} d_{t}\right)$ & $Q_{q q q}^{(1)}$ & $\varepsilon^{\alpha \beta \gamma} \varepsilon_{j k} \varepsilon_{m n}\left[\left(q_{l}^{c}\right.\right.$ & $\left.{ }^{j}\right)^{T} C q_{r}^{\beta}$ & $k]\left[\left(q_{s}^{\gamma m}\right)^{T} C l_{t}^{n}\right]$ \\
\hline$Q_{\text {lequ }}^{(1)}$ & $\left(\bar{l}_{p}^{j} e_{r}\right) \varepsilon_{j k}\left(\bar{q}_{s}^{k} u_{t}\right)$ & $Q_{q q q}^{(3)}$ & $\varepsilon^{\alpha \beta \gamma}\left(\tau^{I} \varepsilon\right)_{j k}\left(\tau^{I} \varepsilon\right)_{m n}$ & {$\left[\left(q_{p}^{\alpha j}\right)^{T}\right.$} & $\left.C q_{r}^{\beta k}\right]\left[\left(q_{s}^{\gamma m}\right)^{T} C l_{t}^{n}\right]$ \\
\hline$Q_{\text {lequ }}^{(3)}$ & $\left(\bar{l}_{p}^{j} \sigma_{\mu v} e_{r}\right) \varepsilon_{j k}\left(\bar{q}_{s}^{k} \sigma^{\mu v} u_{t}\right)$ & $Q_{d u u}$ & $\varepsilon^{\alpha \beta \gamma}\left[\left(d_{p}^{\alpha}\right)\right.$ & $\left.C u_{r}^{\beta}\right]$ & $\left.\left(u_{s}^{\gamma}\right)^{T} C e_{t}\right]$ \\
\hline
\end{tabular}

Table 3: Four-fermion operators.

colour indices in the upper part of Tab. 3. In the lower-left block of that table, colour indices are still contracted within the brackets, while the isospin ones are made explicit. Colour indices are displayed only for operators that violate the baryon number $B$ (lower-right block of Tab. 3). All the other operators in Tabs. 2 and 3 conserve both $B$ and $L$.

The bosonic operators (classes $X^{3}, X^{2} \varphi^{2}, \varphi^{6}$ and $\varphi^{4} D^{2}$ ) are all Hermitian. Those containing $\widetilde{X}_{\mu v}$ are CP-odd, while the remaining ones are CP-even. For the operators containing fermions, Hermitian conjugation is equivalent to transposition of generation indices in each of the fermionic currents in classes $(\bar{L} L)(\bar{L} L),(\bar{R} R)(\bar{R} R),(\bar{L} L)(\bar{R} R)$, and $\psi^{2} \varphi^{2} D^{2}$ (except for $Q_{\varphi u d}$ ). For the remaining operators with fermions, Hermitian conjugates are not listed explicitly.

Counting the entries in Tabs. 2 and 3, we find 15 bosonic operators, 19 single-fermioniccurrent ones, and $25 B$-conserving four-fermion ones. In total, there are 15+19+25=59 independent dimension-six operators, so long as $B$-conservation is imposed.

\section{Comparison with Ref. [1]}

Comparing the $B$-conserving operators in Tabs. 2 and 3 with Eqs. (3.3)-(3.64) of Ref. [1], one finds that 
(i) The only operator missed in Ref. [1] is $Q_{\text {lequ }}^{(3)}=\left(\bar{l}_{p}^{j} \sigma_{\mu v} e_{r}\right) \varepsilon_{j k}\left(\bar{q}_{s}^{k} \sigma^{\mu v} u_{t}\right)$. This fact has been already noticed in Ref. [8] where $\left(\bar{l}_{p}^{j} u_{t}^{\alpha}\right) \varepsilon_{j k}\left(\bar{q}_{s}^{k \alpha} e_{r}\right)=\frac{1}{8} Q_{\text {lequ }}^{(3)}-\frac{1}{2} Q_{\text {lequ }}^{(1)}$ was used instead.

(ii) One linear combination of the three $\varphi^{4} D^{2}$-class operators in Eqs. (3.28) and (3.44) of Ref. [1] must be redundant because this class contains two independent operators only. In fact, presence of all the three operators contradicts correct arguments given in Sec. 3.5 of that paper.

(iii) The number of single-fermionic-current operators in Ref. [1] becomes equal to ours after removing all the 16 operators with covariant derivatives acting on fermion fields (Eqs. (3.30)(3.37) and (3.57)-(3.59) there). All such operators are indeed redundant. This fact has been already discussed in Refs. [14, 13] for most of the cases. Note that removing those operators helps in eliminating multiple assignment of the same operator names in Ref. [1].

(iv) Our use of $\stackrel{\leftrightarrow}{D_{\mu}}$ instead of $D_{\mu}$ in class $\psi^{2} \varphi^{2} D$ does not affect the formal operator counting, but actually reduces the number of terms to be considered. The point is that Hermitian conjugates of our operators with $\stackrel{\leftrightarrow}{D_{\mu}}$ have an identical form as the listed ones, so they do not need to be considered separately. On the other hand, using scalar field derivatives with a positive relative sign (opposite to that in Eq. (2.3)) would give redundant operators only, i.e. linear combinations of the three $\psi^{2} \varphi^{3}$-class terms, EOM-vanishing objects, and total derivatives. This issue has been already noticed in Ref. [15].

(v) Fierz identities (for anticommuting fermion fields) like the following one:

$$
\left(\bar{\psi}_{L} \gamma_{\mu} \psi_{L}\right)\left(\bar{\chi}_{L} \gamma^{\mu} \chi_{L}\right)=\left(\bar{\psi}_{L} \gamma_{\mu} \chi_{L}\right)\left(\bar{\chi}_{L} \gamma^{\mu} \psi_{L}\right)
$$

make 5 out of 29 four-fermion operators in Ref. [1] linearly dependent on the others. For instance,

$$
\left(\bar{l}_{p} \gamma_{\mu} \tau^{I} l_{r}\right)\left(\bar{l}_{s} \tau^{I} \gamma^{\mu} l_{t}\right)=2\left(\bar{l}_{p}^{j} \gamma_{\mu} l_{r}^{k}\right)\left(\bar{l}_{s}^{k} \gamma^{\mu} l_{t}^{j}\right)-Q_{l l}^{p r s t}=2 Q_{l l}^{p t s r}-Q_{l l}^{p r s t}
$$

where the identity

$$
\tau_{j k}^{I} \tau_{m n}^{I}=2 \delta_{j n} \delta_{m k}-\delta_{j k} \delta_{m n}
$$

and Eq. (4.1) have subsequently been used.

As far as the operator names and their normalization are concerned, our notation is close but not identical to the one of Ref. [1]. Taking advantage of the need to modify the notation because of redundant operator removal, we do it in several places where convenience is the only issue. 
The complete list of nomenclature and normalization changes reads:

(i) Unnecessary rationals are skipped in front of $Q_{\varphi G}, Q_{\varphi W}, Q_{\varphi B}, Q_{\varphi}, Q_{l l}, Q_{q q}^{(1)}, Q_{q q}^{(3)}, Q_{e e}, Q_{u u}$ and $Q_{d d}$.

(ii) $T^{A}$ instead of $\lambda^{A}$ are used in $Q_{u G}, Q_{d G}$ and $Q_{\ldots}^{(8)}$.

(iii) Fierz transformation and multiplication by $(-2)$ is applied in our $(\bar{L} L)(\bar{R} R)$ class to avoid crossed colour and Dirac index contractions, and to make the notation somewhat more transparent. In addition, colour-Fierz transformations are applied to linear combinations of the last four operators of this class.

(iv) Operator names are changed in many cases to avoid multiple use of the same symbols, indicate the presence of essential fields, and make the nomenclature more systematic in the four-fermion sector. In particular, the names are modified for $Q_{\varphi W B}, Q_{\varphi \widetilde{W} B}, Q_{\varphi u d}$, as well as in the whole $(\bar{L} R)(\bar{R} L)$ and $(\bar{L} R)(\bar{L} R)$ classes.

One of the reasons for naming our operators with "Q" rather than with "O" is to indicate that many notational details have changed. We have followed Ref. [1] everywhere except for sign conventions for the Yukawa couplings in Eq. (2.1) and inside covariant derivatives (Eq. (2.2)). The latter affects signs of operators in classes $X^{3}$ and $\psi^{2} X \varphi$.

\section{Conclusions}

A simplification of the operator basis by the EOMs can be appreciated by comparing our Tab. 2 that contains 34 entries with Ref. [11] where 106 operators involving bosons are present because no EOM-reduction has been applied. Going down from 106 to 51 with the help of EOMs in Ref. [1] has been a partial success.

The knowledge of the form of effective interactions in the SM is crucial for phenomenological analyses of a possible impact of a broader theory on already known and measured processes. Although the overall list is of certain lenght, only few are important in a specific case. For instance, anomalous $W t b$ couplings that can be well tested at the LHC are described by four operators only $\left(Q_{u W}, Q_{d W}, Q_{\varphi q}^{(3)}\right.$ and $\left.Q_{\varphi u d}\right)[13,15,12]$.

It is interesting to note that if the underlying beyond-SM model is a weakly coupled (perturbative) gauge theory, operators containing field-strength tensors in Tab. 2 cannot be tree-level generated [8]. In consequence, their Wilson coefficients $C_{k}$ are typically $\mathscr{O}\left(\frac{1}{16 \pi^{2}}\right)$. Thus, so long as we are interested in operators with $\mathscr{O}(1)$ coefficients only, as little as 14 entries of Tab. 2 remain relevant. Investigations involving those operators can be found, e.g., in Refs. [9, 16].

\section{References}

[1] W. Buchmüller and D. Wyler, Nucl. Phys. B 268 (1986) 621.

[2] B. Grzadkowski, M. Iskrzyński, M. Misiak, J. Rosiek, JHEP 10 (2010) 85 [arXiv:1008.4884v2]

[3] T. Appelquist and J. Carazzone, Phys. Rev. D 11 (1975) 2856.

[4] H. D. Politzer, Nucl. Phys. B 172 (1980) 349; 
[5] A. A. Belavin, A. M. Polyakov, A. S. Schwartz and Yu. S. Tyupkin, Phys. Lett. B 59 (1975) 85;

[6] S. Weinberg, Phys. Rev. Lett. 43 (1979) 1566.

[7] J. D. Bjorken and S. D. Drell, "Relativistic Quantum Mechanics”, McGraw-Hill Inc., 1964.

[8] C. Arzt, M. B. Einhorn and J. Wudka, Nucl. Phys. B 433 (1995) 41 [hep-ph/9405214].

[9] B. Grzadkowski, Z. Hioki and M. Szafrański, Phys. Rev. D 58 (1998) 035002 [hep-ph/9712357];

[10] J. A. Aguilar-Saavedra, arXiv:1008.3562.

[11] C. N. Leung, S. T. Love and S. Rao, Z. Phys. C 31 (1986) 433.

[12] B. Grzadkowski and M. Misiak, Phys. Rev. D 78 (2008) 077501 [arXiv:0802.1413].

[13] J. A. Aguilar-Saavedra, Nucl. Phys. B 812 (2009) 181 [arXiv:0811.3842].

[14] B. Grzadkowski, Z. Hioki, K. Ohkuma and J. Wudka, Nucl. Phys. B 689 (2004) 108 [hep-ph/0310159];

[15] J. A. Aguilar-Saavedra, Nucl. Phys. B 821 (2009) 215 [arXiv:0904.2387].

[16] B. Grzadkowski and J. Wudka, Phys. Lett. B 364 (1995) 49 [hep-ph/9502415]. 